\title{
MISSION ANALYSIS AND GUIDANCE, NAVIGATION, AND CONTROL DESIGN FOR RENDEZVOUS AND DOCKING PHASE OF ADVANCED REENTRY VEHICLE MISSION
}

\author{
L. Strippoli ${ }^{1}$, P. Colmenarejo ${ }^{1}$, and H. Strauch ${ }^{2}$ \\ ${ }^{1} \mathrm{GMV}$ \\ Isaac Newton, 11, Tres Cantos 28760, Spain \\ ${ }^{2}$ EADS Astrium \\ P.O. Box 286156, Bremen 28361, Germany
}

\begin{abstract}
Advanced Reentry Vehicle (ARV) belongs to the family of vehicles designed to perform rendezvous and docking (RvD) with the International space station (ISS) [1]. Differently from its predecessor ATV (Automated Transfer Vehicle), the ARV will transport a reentry capsule, equipped with a heatshield and able to bring back cargo, experiments, or, as a possible future development, even crew, being this latter scenario very attracting in view of the Space Shuttle retirement. GMV, as subcontractor of EADS-Astrium Germany, is in charge of the RvD and departure mission analysis and GNC (Guidance, Navigation, and Control) design of ARV mission. This paper will present the main outcomes of the study.
\end{abstract}

\section{INTRODUCTION}

Despite of many similarities with ATV, a number of crucial differences influence importantly the ARV rendezvous design, reducing the level of heritage of ATV technologies [2,3]. The most important of these differences is that ARV will dock in the USOS (United States On-Orbit Segment) part of the Station, either at Node 2 longitudinal port or, as a backup option, at Node 2 zenith port. This has a direct impact on the approach/departure/collision avoidance manoeuvre (CAM) trajectories, on the relative sensors that can be used for the approach, and on the dynamics conditions at docking. Consequently, the ARV GNC shall be readapted with respect to ATVs (also to take into account differences in mass, center of gravity, and inertia), and the different RvD figures of merit shall be 
reassessed to verify the fulfillment of ARV requirements. The paper is organized as follows:

- in section 2, the different ARV approach trajectories are analyzed;

- in section 3, the approach followed to design the GNC is shown;

- section 4 presents the ARV Functional Engineering Simulator and some preliminary results obtained with it; and finally,

- section 5 provides the main conclusions of the study.

\section{ANALYSIS OF APPROACH TRAJECTORIES}

Depending on the attitude of the ISS (yaw rotation of $0^{\circ} / 180^{\circ}$ with regard to local vertical and local horizontal (LVLH) reference frame), the approach of ARV can be along $-V$-bar (like ATV) or $+V$-bar. However, independently on final docking direction and ISS attitude, the approach is always based on the following subphases (Fig. 1):

- free drift from $S_{-1 / 2}$ (rendezvous initiation point) to $S_{0}$ (relative global positioning system (RGPS) acquisition point) and from $S_{0}$ to $S_{1}$ (prehoming phase);

- Hohmann transfer from $S_{1}$ to $S_{2}$ (point on $V$-bar at about $3500 \mathrm{~m}$ from ISS); $S_{2}$ side ( $+V$-bar or $-V$-bar) depends on the docking port position in LVLH reference frame (and so on ISS attitude);

- passively safe radial hopping from $S_{2}$ to $S_{3}$ (point on $V$-bar at about $300 \mathrm{~m}$ from ISS, on the same side of $V$-bar with regard to $S_{2}$ ). The exact position of $S_{3}$ depends basically on short range relative sensor operational range;

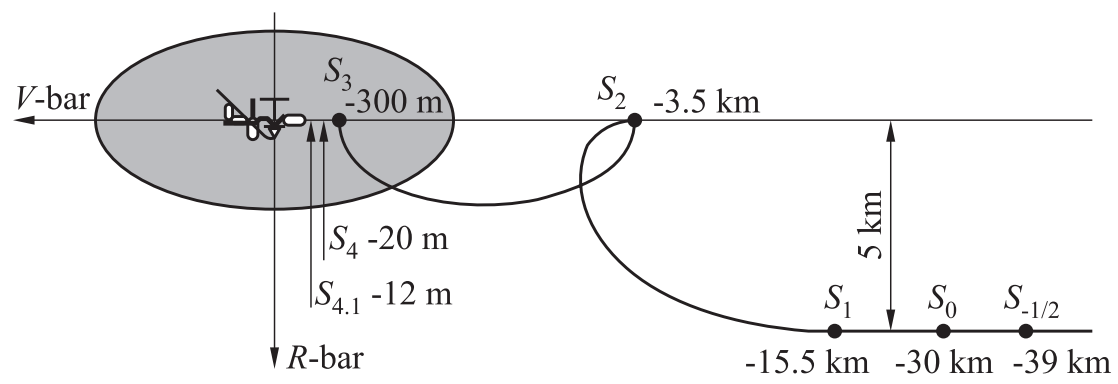

Figure 1 Rendezvous approach points 
- transfer to Forced Motion Starting Point (FMSP). In case of $+/-V$-bar approach, this point coincides with $S_{3}$ and this subphase is missing. Otherwise, a two-point transfer shall be performed to reach the coordinates of the FMSP (that, in general, is not a fixed point in LVLH reference frame because of the ISS attitude motion); and

- forced motion till docking (with station keeping in $S_{4}$ during the approach for waiting go ahead commands). This phase shall be performed in ISS body reference frame in order to take into account the ISS attitude motion.

The attitude, in general, will be yaw-steering till $S_{3}$, in order to maximize the solar power generation guaranteeing, at the same time, the availability of RGPS navigation. During big manoeuvres application, the main thrust axis will be pointed toward the dorsoventral (DV) direction, in order to maximize the efficiency of thrusters actuation. In $S_{3}$, a target pointing will be started in order to point the rendezvous sensors toward the ISS. Finally, in $S_{4}$, where the relative attitude information will be already available, the docking attitude pointing will be commanded.

\section{$2.1+/-V$-Bar Approaches}

Advanced reentry vehicle shall have the possibility to dock along $+V$-bar (US side, nominal ISS configuration) or $-V$-bar (US side, ISS rotated $180^{\circ}$ around its yaw axis). The approach to the Russian part has been discarded due to the traffic foreseen at that docking port.

From trajectory point of view, for the approach along $-V$-bar, there is no reason to introduce relevant differences with respect to the profile successfully adopted for ATV (already shown in Fig. 1). The same phases, the same manoeuvres, and the same checking points (from $S_{-1 / 2}$ to $S_{4}$ ) can be defined also for ARV. The fact that the docking port is in USOS side could influence the GNC performances (different relative sensors, flexible modes, etc.) but not the generic approach profile.

In case of docking along $+V$-bar, different strategies have been investigated, selecting finally the one (shown in Fig. 2) minimizing the differences with respect to the ATV approach. The approach is the same as ATV till point $S_{1}$. The along track coordinate of $S_{1}$ shall be shifted toward the ISS (by increasing the duration of the free drift from $S_{0}$ to $S_{1}$ ), in order to have the point $S_{2}$ on the $+V$-bar side. The rest of the approach from $S_{2}$ to docking is similar to ATV case, except for the fact that the hopping trajectory from $S_{2}$ to $S_{3}$ will be above $V$-bar instead of below. The ARV shall be rotated around its yaw axis in order to properly orientate the RvD face toward the ISS docking port. This can be done at the very beginning of the rendezvous phase (i. e., during the free 


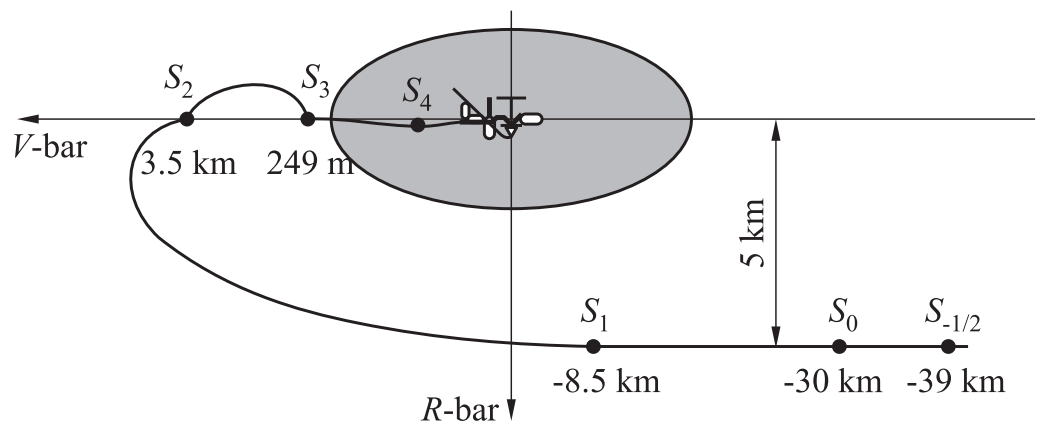

Figure 2 Advanced reentry vehicle $+V$-bar approach profile

drift from $S_{0}$ to $S_{1}$ ), in $S_{2}$ or in $S_{3}$, being the first option, in general, the most reliable because it would increase the reactivity to make available alternative relative measurements in case of RGPS failure. Another option would be following a target pointing in order to point constantly the RvD face toward the ISS. This solution would be necessary in case of intermediate range relative navigation based on optical/infrared sensors, but is not preferable in all the other cases because implies more work for actuators and, so, more power and/or propellant consumption. Furthermore, the target pointing would imply rotating the spacecraft, with consequent possible loss of RGPS and a worse behavior from power generation point of view.

In the approach corresponding to Fig. 2, a nominal propellant consumption of $39.5 \mathrm{~kg}$ (considering a mass of $21,783 \mathrm{~kg}$, a specific impulse of $285 \mathrm{~s}$, and a forced motion velocity of $7 \mathrm{~cm} / \mathrm{s}$ ) is obtained. It includes only the guidance reference manoeuvres and, so, will increase importantly when navigation, control, and, especially, thruster efficiency effects will be included. Furthermore, the final forced motion shall be performed in the ISS body reference frame that adds up an additional propellant consumption with respect to the mentioned nominal value.

\subsection{Non- $V$-Bar Approaches}

The non- $V$-bar approaches will be performed in one of the following situations:

- docking port is the Node 2 zenith port;

- whenever the ISS is not in the $+/-X V V$ attitude, i. e., $+/-Z V V$ or Torque Equilibrium Attitude (TEA); or

- for berthing scenarios. 
In these cases, the FMSP in LVLH frame will be not on $V$-bar and the forced motion direction will be not along $V$-bar. Independently which case is considered, the philosophy of the approach does not change and is described below for the $-R$-bar case and for the TEA case. For the other cases, the only difference is the position in LVLH of the FMSP and the direction of the forced motion. Further, major differences, also from the point of view of propellant consumption, are not foreseen.

Figures 3 and 4 show the complete approach, from $S_{0}$ to docking, with different degrees of zoom, for a $-R$-bar docking scenario. The approach till $S_{3}$ is common to the other approach directions. As shown in Fig. 3, once finished the station keeping in $S_{3}$ (during which the short range relative sensor is acquired and the short range relative navigation converges) a free drift trajectory at a radial coordinate sufficiently high to respect a safety constraint with respect to the highest ISS module $(\sim 35 \mathrm{~m})$. The free drift trajectory will take the ARV up to the FMSP. There are two different ways to reach the free drift trajectory:

(1) by mean of two burns tangential hopping (Hohmann transfer). The problem of this approach is that, in case of a thrust failure in correspondence of the second burn, the resulting trajectory could be not passively safe. In the figure, for example, it is shown, by dash-dotted curve, a free drift deriving from a failure of just $5 \%$ in the second $\Delta V$ modulus; and

(2) by mean of a one-burn radial hopping, sized in order to reach a maximum radial coordinate equal to the free drift trajectory desired height. This manoeuvre inserts the ARV in an elliptic passively safe nondrifting trajectory (fly-around). When the ARV reaches the maximum radial coordinate in the fly-around trajectory, a tangential manoeuvre shall be applied to start the drifting toward the FMSP. The advantage of this strategy is that if the

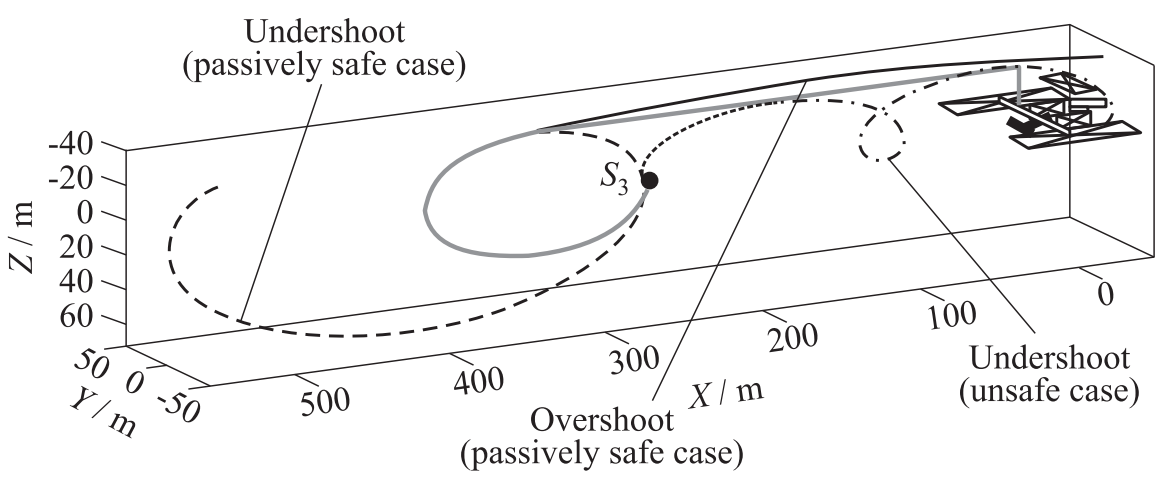

Figure 3 Approach trajectory for $-R$-bar docking (zoom on $S_{3}$ ) 


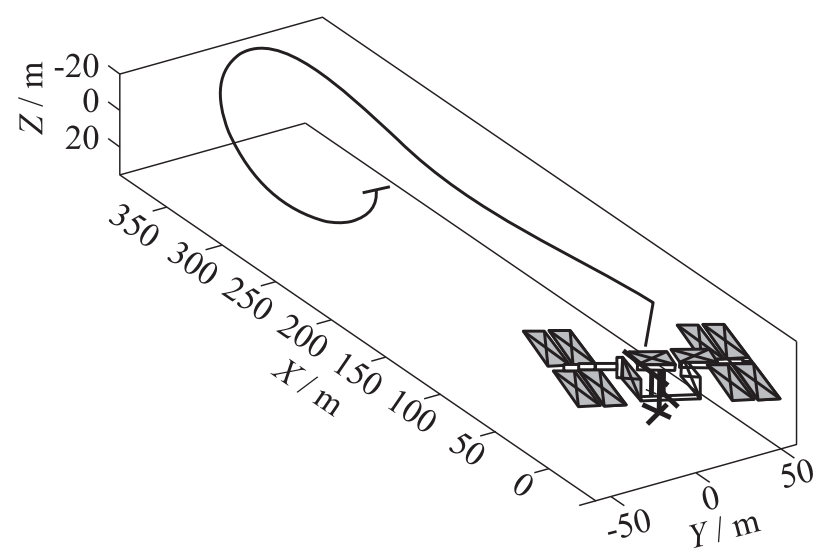

(a)

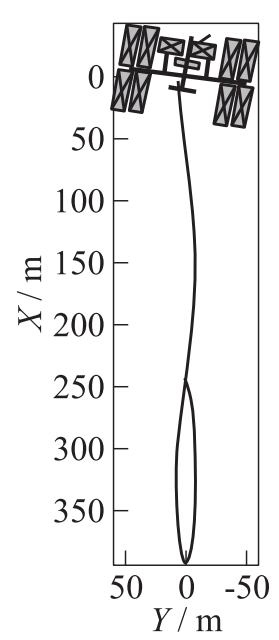

(b)

Figure $4-R$-bar approach trajectory in case of ISS TEA

tangential burn fails, the resulting drift will be passively safe. Figure 3 shows, by dashed and black solid curves, two drifting trajectories, deriving, respectively, from an overshoot and from an undershoot in the tangential $\Delta V$ application.

The nominal propellant consumption is very similar in the two cases $(\sim 43 \mathrm{~kg})$, and this further justifies the utilization of strategy 2 that has no drawback with respect to strategy 1.

In case of performing the docking with the ISS in TEA $\left(+/-15^{\circ}\right.$ with regard to $X V V)$, the approach is not very different from the one described above. It is shown in Fig. 4. The only difference is that the FMSP point will have different coordinates in LVLH reference frame with regard to the nominal $-R$-bar case. The different height will imply a radial hopping manoeuvre with a slightly different $\Delta V$. The difference in along track coordinate will be compensated modifying opportunely the duration of the free drift.

Finally, in general, an out-of-plane coordinate of the FMSP, that in the nominal case is null, will be present. This out-of-plane coordinate can be reached applying, in $S_{3}$, a small out-of-plane manoeuvre (of the order of $1 \mathrm{~cm} / \mathrm{s}$ ): the outof-plane motion generated in this way will be periodic with the orbital period and will permit reaching an FMSP having a whatever out-of-plane component. The propellant consumption in this case is basically identical to the one seen for the nominal $-R$-bar approach (just a very small increment due to the out-of-plane manoeuvre and, possibly, to a slightly longer forced motion). 


\section{GUIDANCE, NAVIGATION, AND CONTROL DESIGN}

A top-down approach has been followed to obtain, starting from the Mission Requirements, the GNC System requirements and, from these, the Guidance, Navigation, and Control functional level requirements, which have been finally used to derive a GNC design. The process, consisting in checking the compliancy of a generic functional design for RvD missions to the specific ARV requirements and tailoring it where these requirements were not fulfilled, has led to the highlevel architecture shown in Fig. 5.

It is organized as a three-layers architecture in which the AMM (Autonomous Mission Management) and the FDIR (Failure Detection, Isolation, and Recovery) are part of a supervisory level, high-level timeline interpreter and safety monitoring performs outer level functionalities and control belongs to the regulatory level, together with navigation, attitude guidance and translational guidance. This kind of architecture is currently the most used for space autonomous systems, both in European and NASA studies, because it provides a good representation of the tasks hierarchy of typical spacecraft.

\section{Guidance, Navigation, and Control Modes}

Conformingly to the GNC requirements, the following modes are defined for the different GNC functions:

- attitude guidance:

- SAG (Safe Attitude Guidance), in charge of commanding an inertial attitude optimizing solar angle on solar arrays or ground station Line of Sight (LoS) angle on communication antenna;

- YSAG (Yaw Steering Attitude Guidance), in charge of pointing axis normal to solar arrays toward Sun while keeping the GPS satellite in view;

- TMAG (Thrust Maximization Attitude Guidance), in charge of pointing the main thrust axis toward the $\Delta V$ direction (typically used for manoeuvres higher than $5 \mathrm{~m} / \mathrm{s}$ );

- T2AG (Target pointing 2 degree-of-freedom (d.o.f.) Attitude Guidance), in charge of pointing the RvD sensor(s) toward the ISS, maximizing, with the third d.o.f., the Sun angle on solar arrays or the ground station visibility; and

- T3AG (Target pointing 3 d.o.f. Attitude Guidance), in charge of aligning the docking ports during the last part of the forced motion (as soon as relative attitude navigation is available); 


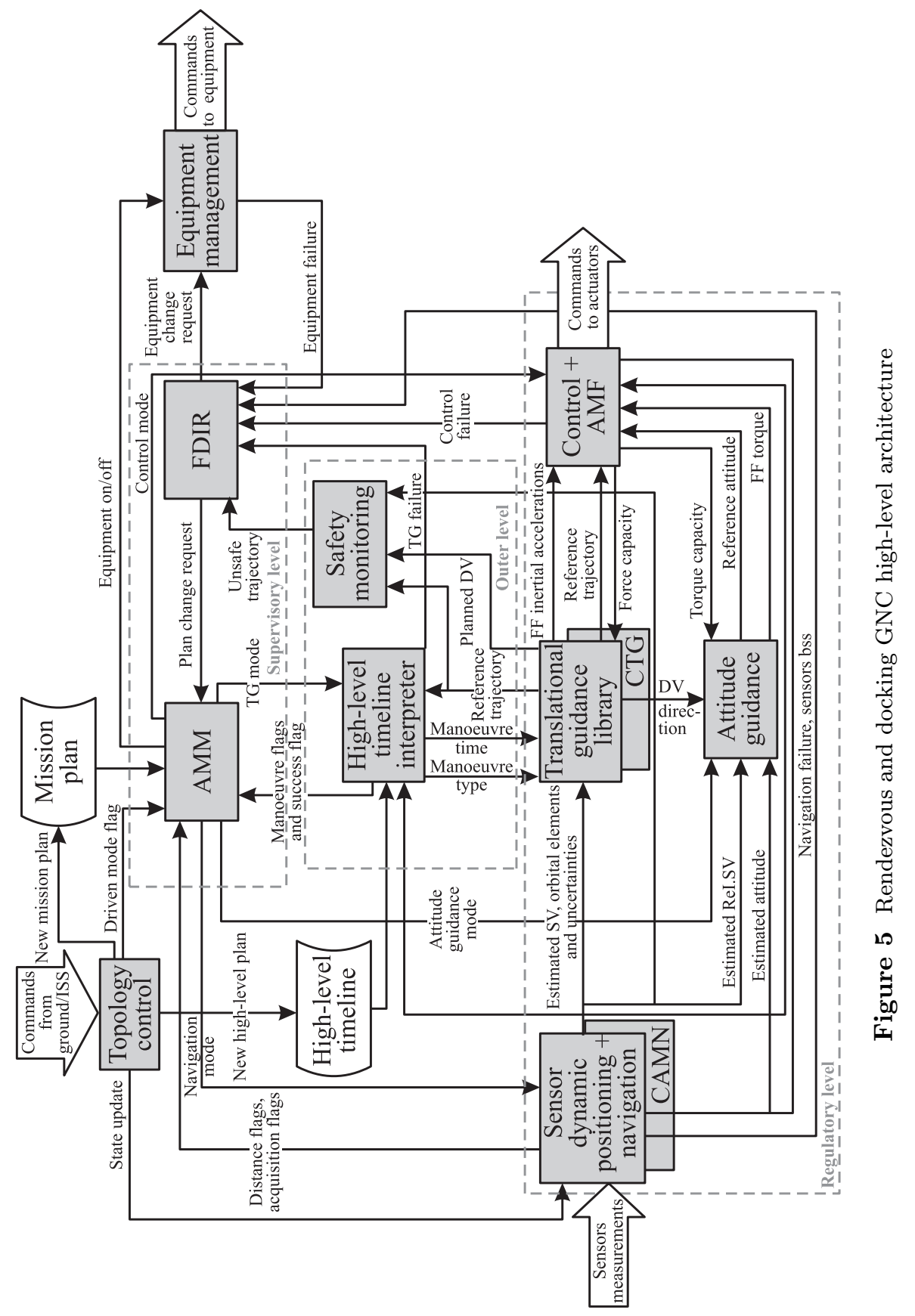


- translational guidance, based on Clohessy-Wiltshire equations [4] with perturbation compensation:

- NOTG (No Translational Guidance): no manoeuvre computation, translational free drift;

- ITG (Impulsive Translational Guidance), in charge of computing and applying manoeuvres during the impulsive phases of the mission (up to FMSP);

- FTG (Forced Translational Guidance), in charge of computing the forced motion leading to docking; and

- CTG (CAM Translational Guidance), in charge of computing CAM manoeuvres;

- navigation, based on Upper Diagonal (UD) Kalman Filters:

- ABSN (Absolute Navigation), in charge of estimating absolute position and attitude;

- RELN (Relative Navigation), in charge of estimating relative position;

- TRDN (Terminal RvD Navigation), in charge of estimating relative position and relative attitude;

- SAFN (Safe Navigation), in charge of estimating the Sun LoS and the angular velocity in ARV body reference frame; and

- CAMN (CAM Navigation), in charge of estimating relative position and relative attitude in the ISS LVLH frame, in case of absence of nominal relative measurements;

- control, based on $H_{\infty}$ synthesis techniques:

- SAPC (Safe Pointing Control): no translation control, thrusters based attitude control with low pointing accuracy but high robustness (in particular, to initial conditions);

- NTRC (Nominal Thrusters-based Control): no translation feedback control (small $\Delta V$, if any, applied in open-loop), thrusters based attitude control with medium pointing accuracy, ability to achieve fast reorientations;

- MBOC (Main Boost Control): no translation control (high $\Delta V$ applied in open loop), thrusters based attitude control with medium pointing accuracy, robustness to propellant sloshes and flexible modes, low agility needs; and

- TRDC (Terminal RvD Mode): translation and attitude control in order to follow both trajectory and attitude guidance; and 
- thrusters management functions:

- 1F3T (3-axis torque with 1-axis force ) for control mode MBOC;

- EF3T (3-axis torque with epsilon disturbing force (if any)) for control modes SAPC and NTRC; and

- 3F3T (3-axis torque and 3-axis force) for control mode TRDC.

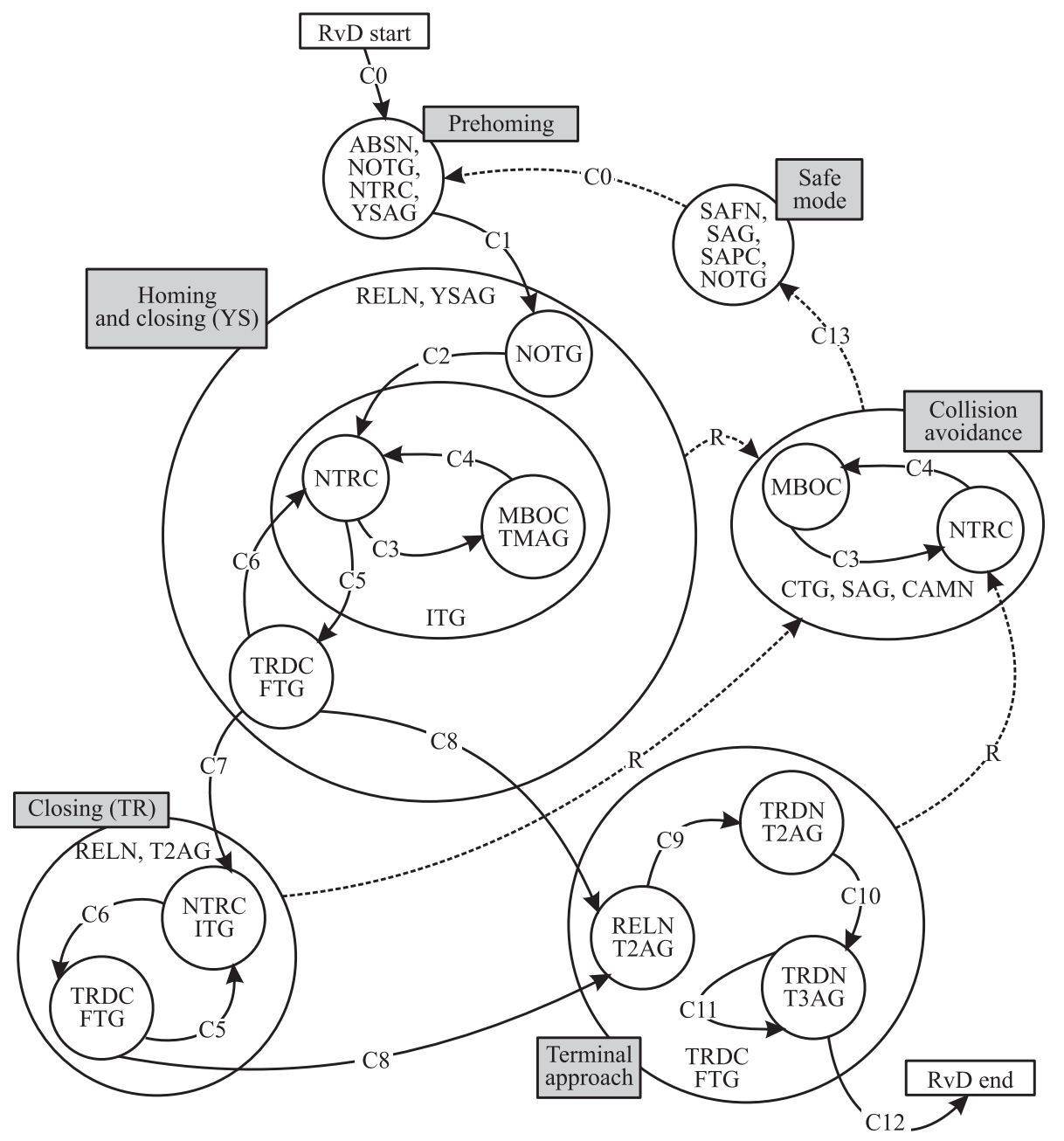

Figure 6 Guidance, Navigation, and Control mode transitions commanded by AMM (left) and transitions conditions (right) 


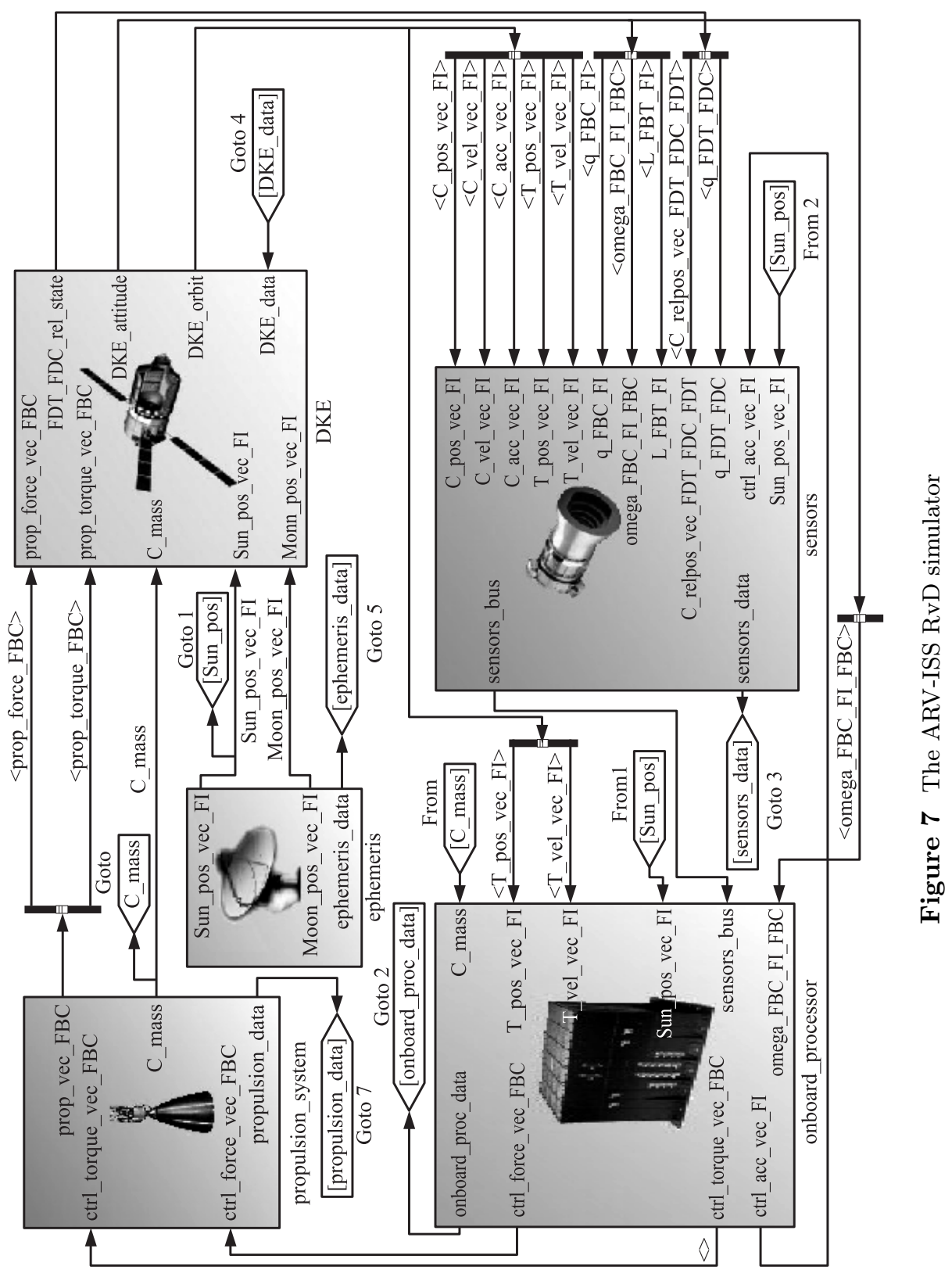


The AMM will be in charge of commanding, based on events and instruction contained in the mission plan, the triggering of the different Guidance, Navigation, and Control modes. In particular, the state transitions shown in Fig. 6 will be implemented in the plan of the ARV RvD.

\section{ADVANCED REENTRY VEHICLE FUNCTIONAL ENGINEERING SIMULATOR AND PRELIMINARY RESULTS}

The 6 d.o.f. ARV Functional Engineering Simulator (FES) (Fig. 7) has been developed with GNCDE framework (a GMV in-house tool for supporting the design and analysis of GNC systems [5]), adapting opportunely the mission template previously developed for the ATV-ISS mission (i. e., modifying sensors, flexible modes, ARV inertia properties, approach trajectory, thrusters properties, control and navigation tuning, etc.).

The ARV-FES will be used to run the different approach scenario described in section 1 . So far, the $+V$-bar scenario has been run, and it has been used to tune the simulator and verifying in a preliminary way the fulfillment of the docking requirements. All the perturbations are considered in the real world (atmospheric, solar radiation pressure, gravitational, third body, fuel slosh, ISS flexible modes, and ISS attitude motion). Figure 8 shows the approach trajectory from $S_{0}$ to docking, while the performances at docking can be seen in Fig. $9 a$, where it can be observed how the 11-centimeter requirement is amply fulfilled.

Figure $9 b$ presents the propellant consumption (real vs. nominal). The nominal propellant consumption is the one coming from translational guidance computation, while the real one (computed integrating thrusters burning times) takes into account different effects such as station keeping control, attitude
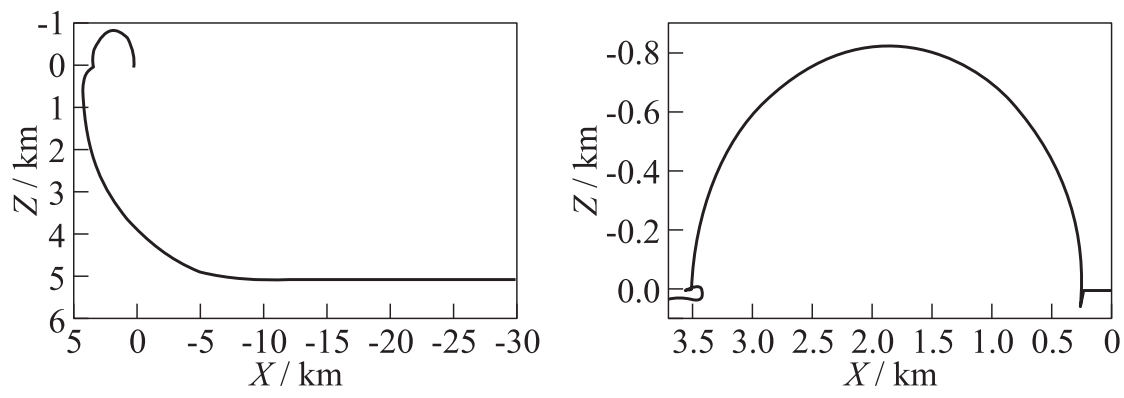

Figure 8 Approach trajectory (zoom on the last meters on the right) 


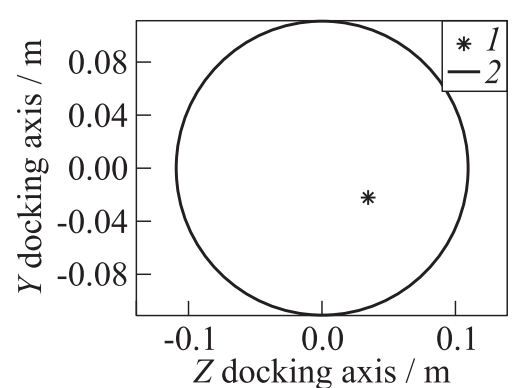

(a)

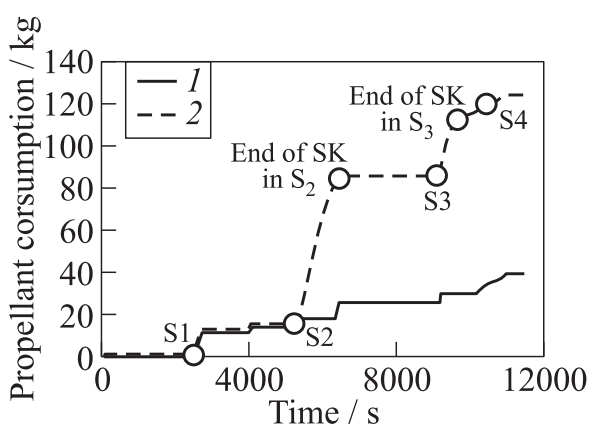

(b)

Figure 9 Docking performances $(a)$ ( 1 - docking performance; and 2 - requirement); and propellant consumption $(b)(1-$ nominal; and 2 - real $)$

control, thruster inefficiency, and correction manoeuvres. The station keeping control has the major contribution to the increment in the propellant consumption.

\section{CONCLUDING REMARKS}

The RvD phase of ARV mission, despite of many similarities with ATV, presents a number of differences reducing the level of heritage of ATV technologies. The GNC design has been adapted to the ARV, by mean of a top-down process tailoring the architecture and the algorithms to the specific mission requirements. The GNC performances are being verified by mean of a functional engineering simulator. More simulations shall be performed, including Monte-Carlos, but the preliminary results seem to confirm the good behavior of the proposed GNC.

The ARV mission is currently in its phase- $A$, close to the System Requirement Review milestone, during which the described GNC will be submitted to ESA evaluation.

\section{REFERENCES}

1. http://www.esa.int/esaMI/ATV/SEMNFZOR4CF_0.html.

2. Ganet, M., I. Quinquis, J. Bourdon, and P. Delpy. 2002. ATV GNC during rendezvous with ISS. DCSSS Conference. 
3. Wartenberg, H., and P. Amadieu. 2002. ATV: Rendezvous with ISS. On Station 11:17-19.

4. Clohessy, W.H., and R.S. Wiltshire. 1960. Terminal guidance system for satellite rendezvous. J. Aerospace Sci. 27(9):653-58.

5. Gandía, F., A. Paoletti, A. Tomassini, M. Sagliano, and F. Ankersen. 2011. GNCDE: Exploiting the capabilities of development environments for GNC design. ESA GNC Conference. 